\title{
Mathematical Modeling of Fluid and Structure Interaction in Ocean Engineering
}

\author{
Yong Liu, ${ }^{1}$ Lin Lu, ${ }^{2}$ Carla Faraci, ${ }^{3}$ and Ming $\mathrm{Zhao}^{4}$ \\ ${ }^{1}$ Department of Ocean Engineering, Ocean University of China, Qingdao 266100, China \\ ${ }^{2}$ Center for Deepwater Engineering, Dalian University of Technology, Dalian 116024, China \\ ${ }^{3}$ Department of Engineering, University of Messina, C/da di Dio, S. Agata, 98166 Messina, Italy \\ ${ }^{4}$ School of Computing, Engineering and Mathematics, Western Sydney University, Penrith, NSW 2751, Australia
}

Correspondence should be addressed to Yong Liu; liuyong@ouc.edu.cn

Received 7 October 2015; Accepted 8 October 2015

Copyright (C) 2015 Yong Liu et al. This is an open access article distributed under the Creative Commons Attribution License, which permits unrestricted use, distribution, and reproduction in any medium, provided the original work is properly cited.

Ocean engineering structures are subject to severe ocean flows, resulting in strong nonlinear fluid and structure interactions. Understanding the physics behind the interactions is of significance to accommodate the increasing demands for designing and building of the ocean engineering structures. The complex interactions between fluid and structures can be simulated by using mathematical models. During the past years, mathematical modeling of fluid and structure interactions in ocean engineering has been greatly developed, and consequently the understanding of physical mechanisms behind it has been remarkably advanced.

This special issue covers the most recent studies on fluid interactions with ocean structures, which includes multiple cylinders, monopile foundation, perforated-wall structures, wave energy devices, and autonomous underwater vehicles. Relevant studies on ocean environment are also included, since they may help in understanding particular features of ocean flows. The common denominator of all these researches in different sectors is the efficient mathematical approach for analyzing the fluid-structure interactions.

Many ocean structures consist of multiple circular cylinders. Flow past circular cylinders is a classical fluid-structure interaction problem, but the mechanism of such a problem is rather complicated. Y. Fu et al. simulated the viscous flow past two tandem circular cylinders at Reynolds number of 100 using an in-house Navier-Stokes equations solver based on the constrained interpolation profile method. The upstream cylinder was forced to perform in-line oscillation while the downstream cylinder was kept stationary. The authors observed different peaks in the spectra of lift forces on both cylinders, along with symmetric $\mathrm{S}$ mode and $2 \mathrm{P}$ mode for the vortex shedding in the lock-in region.

Ocean structures are often bottom-standing or penetrating into the seabed. The wave-structure-seabed interaction may result in seabed instability, which is of great concern in engineering design. C. Zhang et al. developed a 3D integrated numerical model based on a Reynolds-Averaged NavierStokes wave model and a fully dynamic poroelastic seabed model and numerically examined the effects of embedded monopile foundations on the local distributions of pore water pressure, soil stresses, and liquefaction. The authors indicated that the embedded monopole base may lead to a local concentration of soil stress below the two lower corners of foundation, tending to decrease the soil liquefaction depth around foundations.

Ocean structures with perforated thin plates can dissipate the wave energy and significantly reduce the wave forces and slamming loads on the bodies compared to the nonperforated structures. A. Li et al. developed an accurate analytical solution for the wave interaction with perforated thin partial barriers using the multiterm Galerkin method based on linear potential theory. They presented accurate results for both the reflection and transmission coefficients and the wave forces on barriers, which can be used as a reliable benchmark for complicated numerical solutions. X. Ren and Y. Ma developed a 3D viscous numerical wave flume to study 
the cnoidal wave scattering by multiple perforated quasiellipse caissons. The authors found that perforating caisson walls can significantly reduce the wave run-up and wave forces on caissons, and the wave forces acting on caissons become larger at smaller adjacent caisson spacing. However, the effect of adjacent spacing is limited when the ratio of caisson adjacent spacing to caisson width is greater than 3 .

Various wave energy harvesting devices have been proposed and built with the increasing demand for energy. A. J. Garrido et al. developed a simple and efficient analytical model to simulate the dynamic behavior of the energy capture chamber for the oscillating water column device and validated their model by using the experimental data from the Mutriku wave power plant. By using taut wire ropes to connect the damping disk with clump and seabed weights, $Z$. Liu et al. proposed a new heaving buoyancy wave energy device and demonstrated the efficiency and the reliability of the device according to the potential flow theory.

Within the framework of autonomous underwater vehicles, Z. H. Ismail and V. W. E. Putranti proposed a super twisting sliding mode controller with dynamic region concept, which was free of chattering effect. The authors showed that their control method was effective for the robust tracking of an autonomous underwater vehicle and is able to save energy input.

In addition to the above studies, three articles paid special attention to ocean flow problems. L. Zhu and Q. Wang carried out a numerical mesocosm experiment to examine the effect of ocean environment on harmful algal blooms and found that the lack of nutritious supplements can suppress the occurrence of harmful algal blooms in coastal sea. Based on the droplet fractal theory, J.-Y. Liu et al. developed a mathematical model for predicting droplet sauter mean diameter in gas-liquid mist flow and verified their model using experimental data. Q. Zhang et al. used the adjoint method in conjunction with an oceanic Ekman layer model to optimize the vertical distribution of eddy viscosity and the surface wind drag coefficients from pseudo-observations. The authors indicated that the distribution of the vertical eddy viscosity coefficients and the wind stress drag coefficient could be inverted at the same time.

The outcome of all these studies hopefully will contribute to advancing the knowledge of fluid-structure interaction and to providing new incentives and hints for those researchers who are involved in the fascinating field of ocean flows.

\section{Acknowledgments}

We would like to thank all the authors and reviewers for their contributions to this special issue.

$$
\begin{array}{r}
\text { Yong Liu } \\
\text { Lin Lu } \\
\text { Carla Faraci } \\
\text { Ming Zhao }
\end{array}
$$




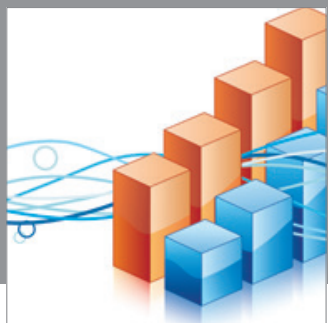

Advances in

Operations Research

mansans

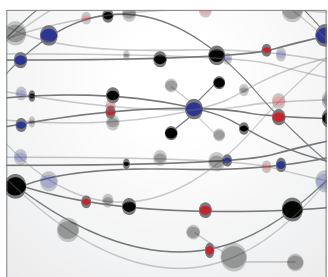

The Scientific World Journal
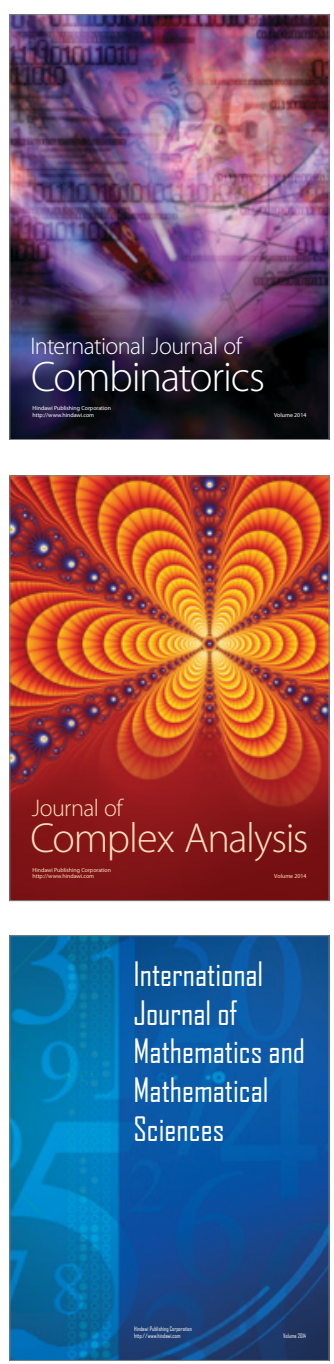
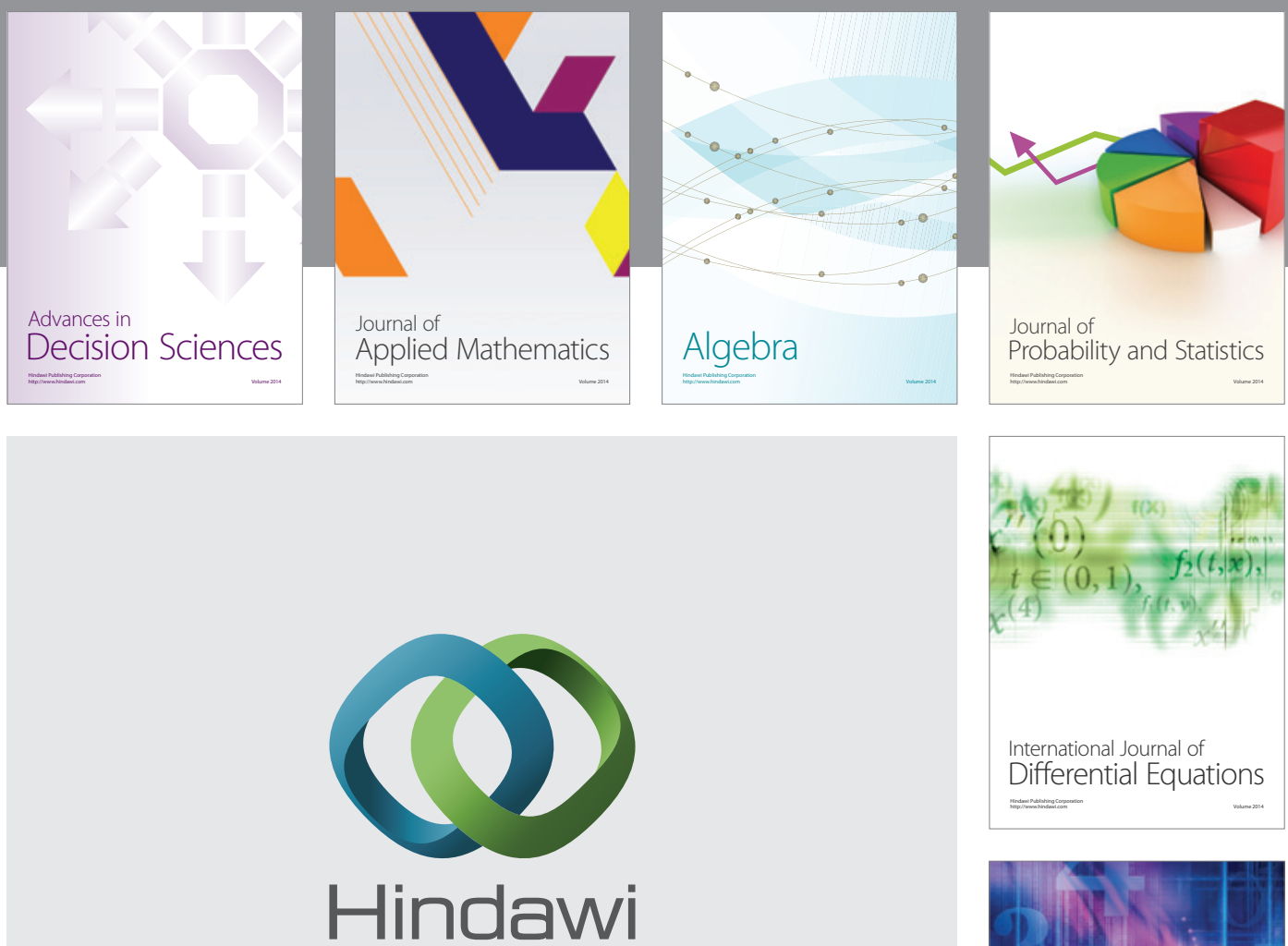

Submit your manuscripts at http://www.hindawi.com
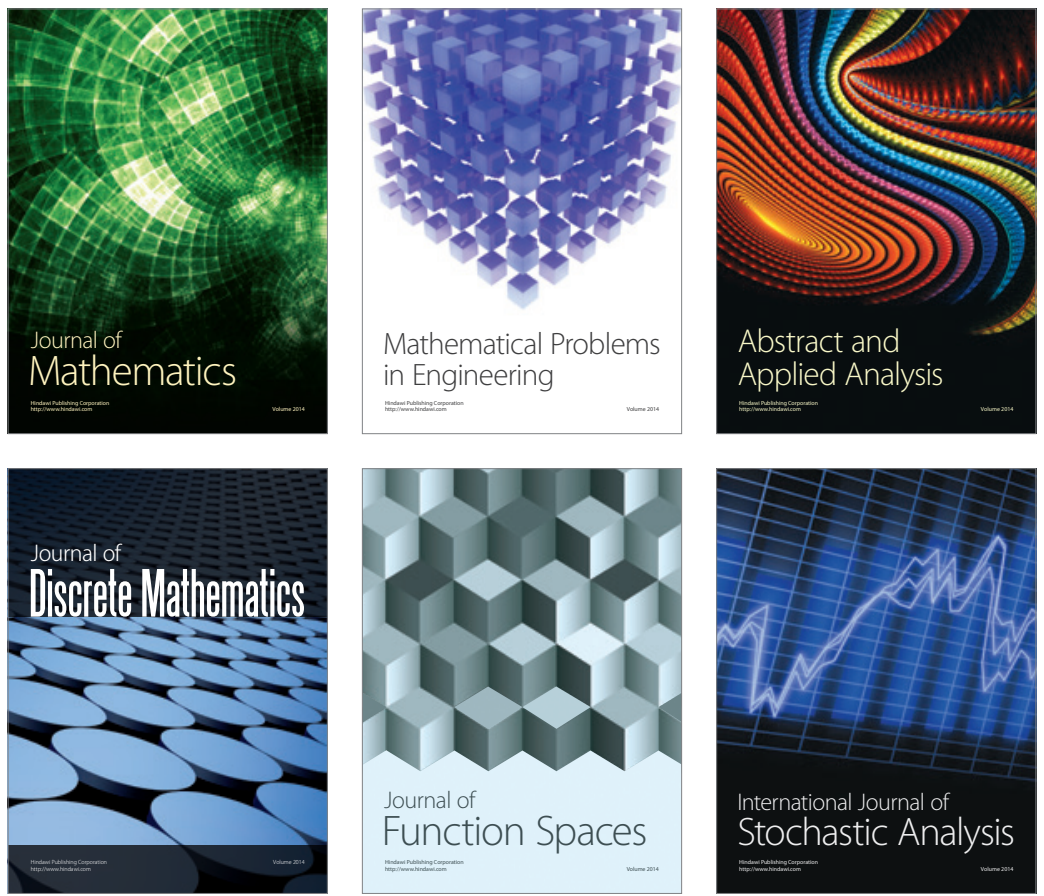

Journal of

Function Spaces

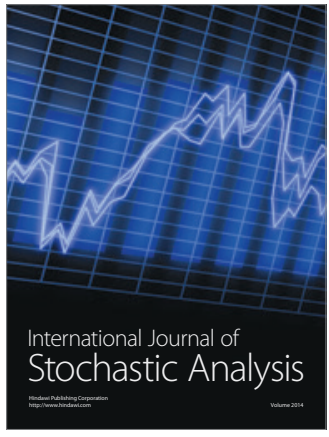

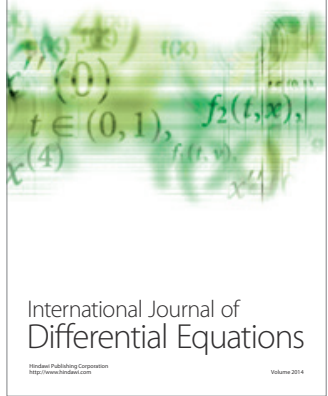
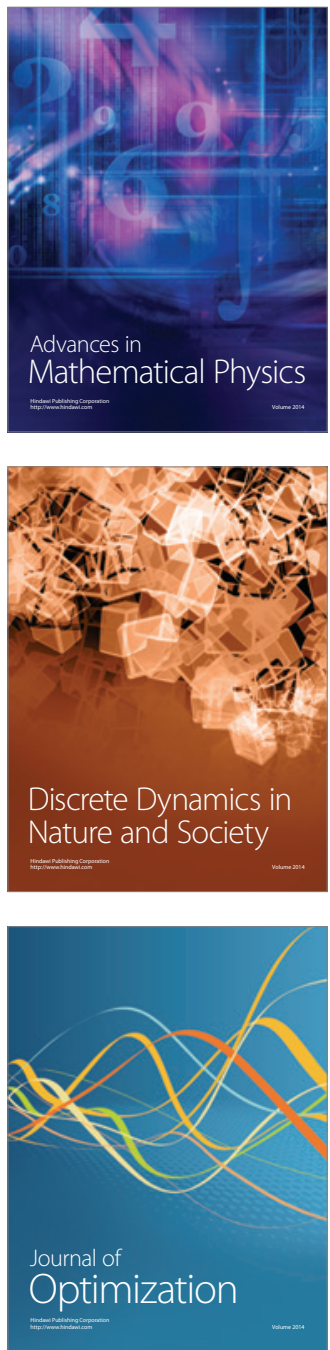